\title{
AUTOMATIC POSITIONING OF SAR GROUND CONTROL POINTS FROM MULTI-ASPECT TERRASAR-X ACQUISITIONS
}

\author{
Sina Montazeri ${ }^{1}$, Christoph Gisinger ${ }^{2}$, Xiao Xiang Zhu ${ }^{1,3}$, Michael Eineder ${ }^{1,4}$ \\ Richard Bamler ${ }^{1,4}$ \\ ${ }^{1}$ Remote Sensing Technology Institute (IMF), German Aerospace Center (DLR) \\ ${ }^{2}$ Chair of Astronomical and Physical Geodesy (IAPG), Technical University of Munich (TUM) \\ ${ }^{3}$ Signal Processing in Earth Observation (SiPEO), TUM \\ ${ }^{4}$ Chair of Remote Sensing Technology (LMF), TUM
}

\begin{abstract}
Geodetic stereo SAR is capable of absolute 3-D localization of natural persistent scatterers (PS)s which allows for Ground Control Point (GCP) generation using only SAR data. The prerequisite for the method to achieve high precision results is the correct detection of common scatterers in SAR images acquired from different viewing geometries. In this contribution, we describe three strategies for automatic detection of identical point targets in SAR images of urban areas taken from different orbit tracks. Moreover, a complete work-flow for automatic generation of large number of GCPs using SAR data is presented and its applicability is shown by exploiting TerraSAR-X high resolution spotlight images over the city of Oulu, Finland and a test site in Berlin, Germany.
\end{abstract}

Index Terms - Geodetic stereo SAR, Ground Control Point, synthetic aperture radar, TerraSAR-X

\section{INTRODUCTION}

Imaging geodesy and geodetic stereo SAR are relatively new techniques which aim at precise absolute positioning of point targets in SAR images in 2-D and 3-D, respectively [1,2]. The latter solves the range-Doppler equation system which relates the satellite position and velocity to the 3-D position of the target on the ground. The method requires the range and azimuth time coordinates of the target extracted from SAR images of the same scene which have to be corrected for atmospheric path delays and the signals of the dynamic Earth. Additionally, the state vector of the satellite at the time of each acquisition is expressed by a polynomial model enabling the method to deliver the 3-D Cartesian coordinates of the target by means of least squares parameter estimation [2]. The precision of the estimated 3-D coordinates depends on the Signal-to-ClutterRatio (SCR) of the target, the precision of the external radar timing corrections, the difference in the viewing geometries and the number of acquisitions.

In terms of viewing geometry, a target can be visible either from same-heading orbits only, i.e. exclusively ascending or descending orbits, or also from cross-heading orbits, which include combinations of ascending and descending orbits. Conceptually, a target localized from the latter is favorable because of the more robust intersection geometry when compared to the former. However, the rare occurrence of common scatterers visible from cross-heading tracks as well as the challenging task of automatically detecting such targets limits the applicability of the geodetic stereo SAR method for localization of large number of cross-heading PSs.
This paper describes a fully automatic algorithm for detection and localization of large number of natural PSs in SAR images of urban areas. The main focus is put on the identification part. Additionally, the applicability of the algorithm is demonstrated by exploiting TerraSAR-X high resolution spotlight images over the city of Oulu, Finland and a test site in Berlin, Germany.

\section{DETECTION OF IDENTICAL SCATTERERS IN MULTI-ASPECT SAR IMAGES}

Detection of identical PSs from SAR images acquired with different viewing geometries is a challenging task. This is because of the geometrical distortions of SAR images due to the oblique viewing geometry and more importantly the presence of speckle. Moreover, relevant to urban areas captured by SAR sensors, which is the focus of this study, the backscattering mechanism is highly complex because of existence of several phase-centers close to each other. Therefore, identical PS matching becomes even more difficult in urban areas. In the following, we describe three strategies to overcome the mentioned problem. Each method has its pros and cons which are described briefly.

\subsection{PSI multitrack point cloud fusion}

In [3], a method for geometrical fusion of multitrack PSI point clouds has been proposed. The fusion algorithm, which is based on the geocoded PS point cloud of each geometry, consists of three major parts, namely: 1) generation of initial point correspondences, 2) restricted least squares adjustment to minimize the distance between assumed identical points visible from different viewing geometries, and 3) adding a range-dependent shift to all PSs using the result of the previous step for the final registration. For a detailed description of the algorithm the reader is referred to [3].

Since we are interested in the detection of large number of point correspondences, only the first part of the algorithm is relevant. This coarse registration is based on cross-correlation of geocoded PS point clouds, after a projection on a regular grid, in the $x y-, x_{-}$ and yz-plane. For the same-heading tracks, this method typically generates, on average, 200 to 2000 point correspondences per $\mathrm{km}^{2}$ depending on how densely constructed is a city which directly affects the total number of PSs in each point cloud. The benefit of the method is that it can provide point correspondences even on buildings and areas with complex scattering mechanisms in SAR images. Therefore, a large number of potential PS pairs can be obtained which cover the entire scene. The downside of the method is 
that it is usually applicable only for same-heading PS point clouds. Consequently in terms of localization precision with the subsequent stereo SAR, the relative error in the cross-range is larger than the error for range and azimuth components. Also not all the initial PS correspondences are useful candidates for stereo SAR as the registration is performed within the limits of the PSI 3-D localization quality for which PS pairs with distances of up to five meters are detected. Finally, the precise detection of GCP candidates relies on a large number of SAR images in the stacks.

\subsection{Template matching on optical data}

Given the availability of suitable remotely sensed optical data, one can detect candidate objects from optical images which are probable to be visible in SAR images from different viewing geometries. In urban areas, scatterers which are good candidates to be visible from both same-heading and cross-heading tracks are usually stemmed from lamp poles or other cylindrical objects that are vertically oriented towards the sensor. Therefore, the basic idea when using optical data for the aid of GCP identification is to detect lamp poles and match the detected objects to the corresponding bright points in SAR images.

The method identifies lamp poles based on their distinctive shadows in optical images using a template matching scheme [4]. Based on this, the template is extracted from the optical data after conversion to grayscale image, negative intensity transformation, histogram equalization and sharpening using high boost filtering. The template is then convolved with the reference image and the Normalized Cross Correlation (NCC) map is calculated. After thresholding on NCC values, the detected points are geocoded and clustered by mean shift clustering using a flat kernel.

The advantage of the method is that it is quite straightforward to implement and it provides identical scatterers that are visible from cross-heading orbits. The disadvantage is that for reliable detection of lamp poles, the spatial resolution of the optical image should be in the sub-decimeter regime. Moreover, the method is highly prone to detecting other linear structures as shadow of the lamp poles.

\subsection{Road network data}

In urban areas, the cylindrical objects of our interest (lamp poles, road signs, traffic lights, etc.) are typically located along the roads. Therefore, with the availability of geospatial road data, either obtained from OpenStreetMap or country-specific geoportals, and the projection of such maps on the SAR images, one can search for bright points in the neighborhood of the road data. The extraction of the candidate bright point is based on calculating the amplitude dispersion index (ADI) in the co-registered stack of SAR images [5]. The pixel with the lowest ADI in a search window centered on the road data node is chosen as potential candidate. If several pixels with low ADI are available in one neighborhood, the ones with ADI lower than a certain threshold are chosen.

The advantage of the method is the detection of a large number of candidates from cross-heading geometries. Also the external road data is freely accessible for most of the locations. The disadvantage is that a co-registration on one master should be carried out for each stack.

\section{AUTOMATIC GCP POSITIONING: PROCESSING CHAIN}

The overall processing chain for automatic GCP positioning consists of the following parts:

A ) identification of identical scatterers visible in multi-aspect SAR images.

B ) radar-coding of the scatterer locations into the complex single-look slant-range SAR images.

C ) precise extraction of scatterers' azimuth and range times from SAR images at sub-pixel level.

D ) scatterer visibility check throughout the images and removal of outliers from time series of phase noise.

E ) correction of the radar timings for perturbing signals.

$\mathrm{F}$ ) estimation of the 3-D absolute coordinates of the scatterers.

Part A was already covered in section 2. Part B is concerned with solving the range-Doppler equations by knowledge of the approximate 3-D coordinates of the scatterer on the ground and the satellite state vector [6]. Part $\mathrm{C}$ involves the point target analysis (PTA) for detecting the scatterer response in azimuth and range with subpixel sensitivity [6]. In part D, the phase noise which is calculated based on the estimated SCR from the previous step, serves as an indicator for outlier detection and visibility check of the scatterer in the SAR images. Subsequently, in part E the extracted timings are corrected for atmospheric, geodynamic and satellite calibration effects to obtain absolute radar timings [1]. Finally, in part F, the scatterer timings from different viewing geometries are combined to estimate the absolute 3-D coordinates of the target with the stereo SAR method [2].

\section{EXPERIMENTAL RESULTS}

\subsection{Data description}

In this study, four stacks of TerraSAR-X high resolution spotlight SAR images over the city of Oulu are exploited from which two were acquired from descending orbits and two were acquired from ascending orbits. Furthermore, for the test site in Berlin two stacks of cross-heading TerraSAR-X images, also in spotlight mode, were used. Complementary to the SAR data, for Berlin an aerial image with ground distance spacing of $7 \mathrm{~cm}$ and a geocoding accuracy in the decimeter regime was used [7], whereas in Oulu the road map data was freely accessed from National Land Survey of Finland (http://www.maanmittauslaitos.fi/en).

\subsection{Stereo PS candidate identification}

In this subsection, the different methods which were described in Section 2 are employed for finding suitable stereo PS candidates in multi-aspect SAR images. The first example includes the identification of PS correspondences from the same-heading tracks of Oulu. Fig. 1a shows the geocoded PS point clouds from the two descending orbits, visualized in green. The red points represent the identified PS pairs from the PSI fusion algorithm. The total number of the correspondences is approximately 32000 and the Euclidean distance between the matched PSs varies from 1.5 to 5 meters. In order to reduce the number of PS correspondences to the ones with higher quality and closer distance, as well as to preserve the homogeneity of the distribution, a regular grid was imposed on the point clouds. Inside the $10 \mathrm{~m} \times 10 \mathrm{~m}$ grid, the PS pairs which were closer together 
and had lower ADI values were selected to reduce the number of potential pairs from 32000 to 10000 . The comparison between PS pairs before and after reduction can be seen in a zoomed-in area in Fig. 1b and Fig. 1c, respectively.

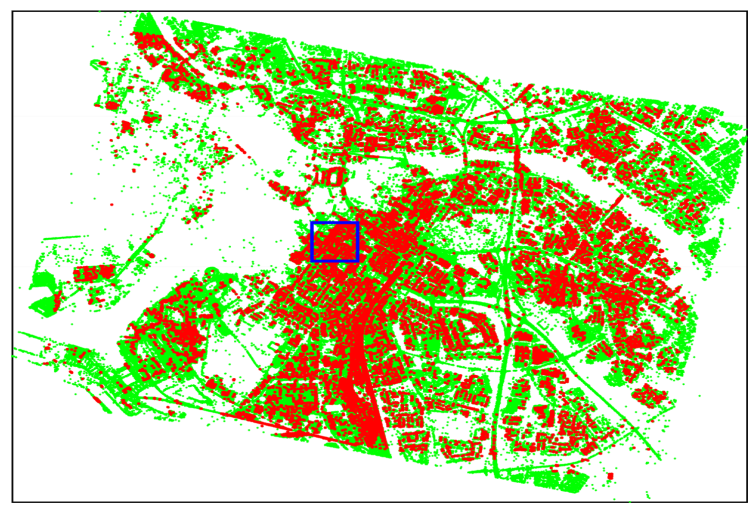

(a)

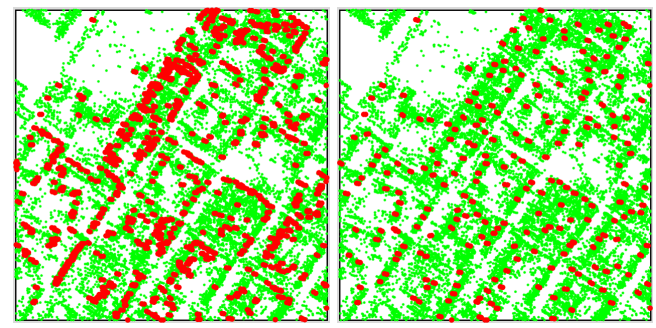

(b)

(c)

Fig. 1: PS correspondence detection based on the PSI fusion algorithm for same-heading PS point clouds in Oulu, Finland.

The second example deals with the identification of PS correspondences from the cross-heading tracks of Berlin. It is seen from Fig. 2a that in an optical image lamp poles can be recognized by their shadows on the ground which can be used as detection template. The NCC map is calculated by template matching and the pixels with NCC values above 0.6 are selected as lamp poles. Subsequently, the UTM coordinates of the pixels are clustered which reduces the pixels from 2030 to 43 that presumably represent the lamp poles. Fig. 2b shows the detected lamp poles in UTM coordinates as black markers. Fig. 2c and Fig. 2d show the radar coded lamp poles on descending and ascending SAR images which are represented by the bright points inside the green circles.

The final example demonstrates the identification of PS correspondences from same and cross-heading tracks using the freely available road data of Oulu. The results displayed in Fig. 3 show the detected bright points, presumably all lamp poles, which are visible in all four geometries for a small area in Oulu. The track type, heading angle $\alpha$ and average incidence angle $\theta_{i n c}$ are also mentioned. All the correspondences are closer than three meters from each other in the UTM coordinate system. The total number of such scatterers is 107 in the entire area of Oulu.

\subsection{Candidate visibility check and data trimming}

After identification of common scatterers, radar coding and precise extraction of their timings in all the available SAR images from the different geometries, the time series of phase noise are analyzed.

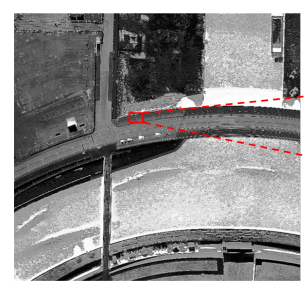

(a)

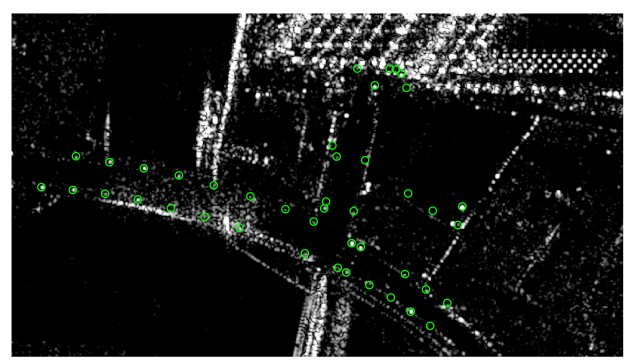

(c)

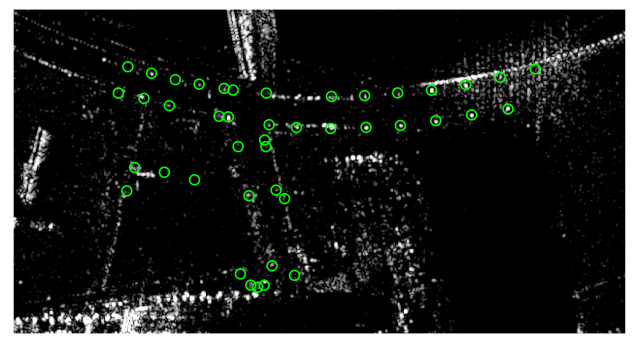

(d)

Fig. 2: PS correspondence detection based on high resolution optical data for cross-heading tracks in Berlin.
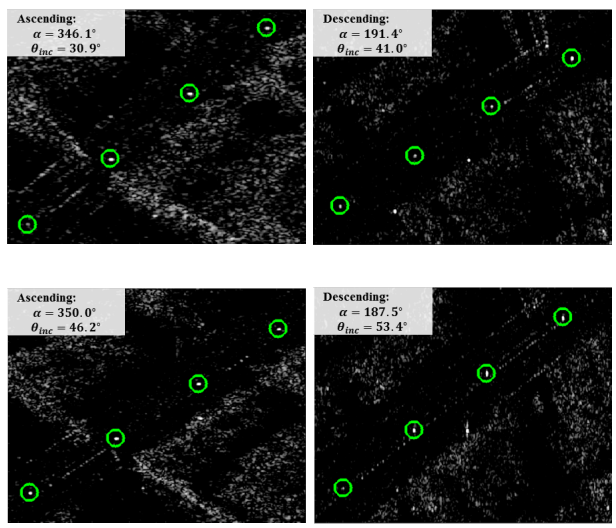

Fig. 3: PS correspondence detection based on road network data for same and cross-heading tracks in Oulu.

The example given in Fig. 4 shows the time series for the phase noise of two stereo PS candidates sorted according to temporal pro- 
Table 1: Averaged statistics based on the least squares estimated 3-D coordinate standard deviations in Oulu. The letters A and D stand for ascending and descending geometries, respectively. The sample mean and standard deviation are denoted by $\mu$ and $\sigma$, and $s_{[E N H]}$ represent the local coordinates standard deviations within $95 \%$ confidence level

\begin{tabular}{cccccccc}
\hline Geometry & Nr. Scatterers & $\mu_{\mathrm{s}_{\mathrm{E}}}[\mathrm{cm}]$ & $\mu_{\mathrm{s}_{\mathrm{N}}}[\mathrm{cm}]$ & $\mu_{\mathrm{S}_{\mathrm{H}}}[\mathrm{cm}]$ & $\sigma_{\mathrm{s}_{\mathrm{E}}}[\mathrm{cm}]$ & $\sigma_{\mathrm{S}_{\mathrm{N}}}[\mathrm{cm}]$ & $\sigma_{\mathrm{S}_{\mathrm{H}}}[\mathrm{cm}]$ \\
\hline AA & 565 & 17.73 & 5.04 & 15.87 & 11.98 & 2.63 & 11.09 \\
DD & 1417 & 15.08 & 3.80 & 16.71 & 10.38 & 2.10 & 11.30 \\
AD & 24 & 2.26 & 2.50 & 1.75 & 0.99 & 1.11 & 0.83 \\
ADAD & 43 & 1.17 & 1.40 & 1.12 & 0.42 & 0.55 & 0.37 \\
\hline
\end{tabular}

gression. The unit of the y-axis in the scatter plots is in radians. It is seen that since the distribution of the phase noises is skewed, removal of outliers based on statistical measures such as mean or median is meaningless. Therefore, we use a method based on adjusted box-plot which considers the skewness of the distribution for outlier removal [8]. In the two examples the outliers are shown within the red rectangles and are identified automatically. It is important to note that after the outlier removal, the scatterer will be removed from the timing observations if the scatterer phase noise is still higher than 0.5 radians in any of the data takes.
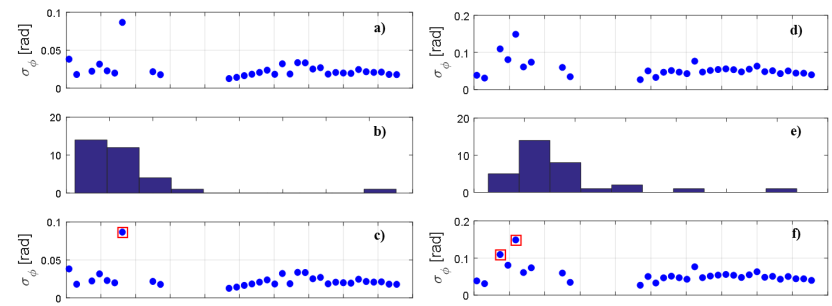

Fig. 4: Examples of phase noise time-series for robust outlier removal. The y-axes of the scatter plots show phase noise values in radians.

\subsection{PS positioning results}

The PS raw timings from the previous step are the input for corrections [1] and the subsequent stereo SAR [2]. Apart from the 3-D coordinates, the stereo SAR method provides a posteriori estimate of standard deviations for both observations, i.e. the range and azimuth timings, as well as the estimated coordinates which can be used to carefully determine and remove outliers [2]. Initially, a two-step outlier removal procedure is applied. First the observations with residuals larger than 1.5 times the resolution of high resolution spotlight images are eliminated. After recomputing, observations with residuals larger than two times the standard deviation are further removed. Additionally, to remove the PSs which are not ideal for stereo SAR due to wrong correspondence matching caused by several scatterers being too close, a third step of data cleaning is performed to exclude PSs with azimuth residuals higher than $20 \mathrm{~cm}$.

Tab. 1 reports on the averaged quality of the estimated 3-D coordinates in Oulu categorized based on the geometric configuration. The results of Berlin are not discussed due to the observed inconsistencies in the positioning outcome which happened because of four major processor update during the analyzed period. From Tab. 1, it is seen that the averaged precisions are smaller than two decimeters for all the cases. As it was expected, the localization quality boosts as the difference in the viewing geometries becomes larger which is the case for cross-heading and quad geometry configuration.

\section{CONCLUSIONS}

In this paper we described a processing chain for absolute localization of opportunistic PSs where the focus was put on the identification of identical scatterers from multi-aspect SAR images. It has been shown that lamp poles are good candidates to be even visible from two pair of cross-heading geometries while in same-heading geometries scatterers originated from buildings could also be processed with stereo SAR. The method allows for identification and positioning of large number of GCPs which have applications such as: detection of large magnitude ground motion, supporting phaseunwrapping in InSAR time series techniques and being used as tie points for registration of optical remotely sensed images.

\section{REFERENCES}

[1] M. Eineder, C. Minet, P. Steigenberger, X. Y. Cong, and T. Fritz, "Imaging Geodesy - Toward Centimeter-Level Ranging Accuracy With TerraSAR-X," IEEE Transactions on Geoscience and Remote Sensing, vol. 49, no. 2, pp. 661-671, Feb. 2011.

[2] C. Gisinger, U. Balss, R. Pail, X. X. Zhu, S. Montazeri, S. Gernhardt, and M. Eineder, "Precise Three-Dimensional Stereo Localization of Corner Reflectors and Persistent Scatterers With TerraSAR-X," IEEE Transactions on Geoscience and Remote Sensing, vol. 53, no. 4, pp. 1782-1802, Apr. 2015.

[3] S. Gernhardt, X. Y. Cong, M. Eineder, S. Hinz, and R. Bamler, "Geometrical Fusion of Multitrack PS Point Clouds," IEEE Geoscience and Remote Sensing Letters, vol. 9, no. 1, pp. 3842, Jan. 2012.

[4] S. Montazeri, X. X. Zhu, U. Balss, C. Gisinger, Y. Wang, M. Eineder, and R. Bamler, "SAR ground control point identification with the aid of high resolution optical data," in Geoscience and Remote Sensing Symposium (IGARSS). IEEE, Jul. 2016, pp. 3205-3208.

[5] A. Ferretti, C. Prati, and F. Rocca, "Permanent scatterers in SAR interferometry," IEEE Transactions on Geoscience and Remote Sensing, vol. 39, no. 1, pp. 8-20, Jan. 2001.

[6] I. G. Cumming and F. H.-c. Wong, Digital processing of synthetic aperture radar data: algorithms and implementation, ser. Artech House remote sensing library. Boston: Artech House, 2005.

[7] H. Hirschmuller, "Stereo Processing by Semiglobal Matching and Mutual Information," IEEE Transactions on Pattern Analysis and Machine Intelligence, vol. 30, no. 2, pp. 328-341, Feb. 2008.

[8] M. Hubert and E. Vandervieren, "An adjusted boxplot for skewed distributions," Computational Statistics \& Data Analysis, vol. 52, no. 12, pp. 5186-5201, Aug. 2008. 